\title{
Eye movements do not cause induced motion
}

\author{
PAUL H. SCHULMAN \\ SUNY College of Technology, Utica, New York 13502
}

\begin{abstract}
An eye-movement theory of induced motion was compared to one based on object-relative displacement as modified by adjacency. Two lines moving in opposite directions served as the inducing stimuli, and a stationary target could be placed in one of three positions: adjacent to the top line, midway between the lines, or adjacent to the bottom line. In the fixation condition, the observer was instructed to fixate the stationary target during each of the three target placements. In the tracking condition, the subject was instructed to follow with his eyes one of the moving lines when the target was in each of its placements. Adjacency determined the results in both conditions, suggesting that eye movements do not cause induced motion.
\end{abstract}

Eye movements, or a tendency to make eye movements, are essential to at least two explanations of induced motion. One explanation posits that the observer tends to track the moving surround, causing the stationary target to displace across his retina (Kaufman, 1974, p. 386). Since, according to this theory, the perceptual system fails to monitor pursuit eye movement, the retinal displacement of the stationary target results in perceived motion. However, Brosgole, Cristal, and Carpenter (1968) found no evidence for this in a direct test. They monitored the observer's eye movements during induced motion while he was attempting to fixate the stationary target. The inducing surround moved $10.3 \mathrm{deg}$, and the mean extent of induced motion, measured separately, was $2.62 \mathrm{deg}$. The mean drift of the six observers' eyes, however, was only $.09 \mathrm{deg}$ in the same direction as the motion of the surround, and the eyes of two observers drifted against the frame. Brosgole et al. concluded that there was no relation between eye movements and induced motion.

Bruell and Albee (1955), however, proposed a theory of egocentric localization which can account for induced motion without an actual eye movement occurring. Each retinal locus indicates a given visual direction; each has a local sign. When the eyes move, eye position is taken into account, which results in a change in the local sign. According to these authors, local signs change only during voluntary eye movements; if an involuntary or reflexive eye movement is made, the local signs remain the same. For instance, if an observer with an afterimage on his fovea was looking straight ahead, and then voluntarily turned his eyes to the right, the afterimage would appear to move to the right; i.e., the local

This research was part of the author's PhD dissertation submitted to the Department of Psychology, the Graduate Faculty of the New School for Social Research. sign of the fovea would now indicate "right" where formerly it had indicated "straight ahead." If this observer pushed his eye to the right with his finger-which would be equivalent to an involuntary eye movement-though the fovea would move to the right, the afterimage would appear stationary; i.e., the local sign of the fovea would remain "straight ahead." (But see Festinger \& Easton, 1974, and Festinger, Sedgwick, \& Holtzman, 1976, who argue that smooth pursuit eye movements, even when voluntary, are not taken into account and so local signs do not change.)

Bruell and Albee assumed that there was an involuntary tendency to follow the surround during induced motion, a tendency similar to pushing one's eye. This is the tendency Brosgole et al. sought but could not find, perhaps because the observer was instructed to fixate the target; in fixating, he balanced this tendency by voluntarily innervating his eye muscles to turn in the direction opposite the surround. The net result was no eye movement. This voluntary tendency, however, changed the local sign of the fovea (as well as of the rest of the retina) and the target appeared to move against the surround. Thus, according to this theory, an actual movement of the eye is not necessary to account for induced motion.

These two eye-movement theories, while different, are complementary. If the eye does not resist the tendency to follow the surround, then the unaccounted for retinal slip of the target could generate the induced motion. If the eye does resist the tendency to follow the surround, then the accounted for voluntary eye movement and resulting change in local sign could generate the induced motion.

A problem arises, however, if the observer intends to follow the surround. The retinal displacement of the enclosed target could account for induced motion only if the perceptual system does not monitor pursuit eye movements. But if it does and if 
the entire eye movement is taken into accountif the change in local sign equals the extent of eye movement-Bruell and Albee would have to argue that no induced motion should be seen. However, as Mack and Herman (1972) have shown with a single point, tracking distance is perceptually underestimated. Furthermore, Mack and Herman (1973) have also shown that this underestimation can account for the Filhene illusion, an illusion in which the background appears to move in the opposite direction of a tracked point. If their argument applies to induced motion, the change in local sign will be less than the extent of eye movement, and this difference will be made up by the perception that the target is moving in the direction opposite the surround. If this interpretation of Bruell and Albee's theory is correct, then both their theory and Kaufman's would make the same prediction when the observer intends to track the surround: The target will appear to move opposite the tracked surround.

In the experiment below, these eye-movement theories were compared to a theory in which objectrelative displacement, as modified by adjacency, determines induced motion. Gogel and Koslow (1971, 1972) have shown that as a stationary spot becomes more adjacent to an inducing surround, the amount of induced motion increases, and if there are two inducing surrounds moving in opposite directions, the more adjacent one determines the direction of induced motion. Using points of light, Gogel (1974) also showed that adjacency determined which of two relative motion cues would be more effective. In this experiment, Gogel did not, however, control for eye movements.

These two theories of induced motion were tested in an experiment in which there were two inducing objects. The inducing objects were horizontal lines, one placed above the other, and they moved horizontally, 180 deg out of phase. The observer tracked either the top or bottom line and the stationary target was placed adjacent to the top line, to the bottom line, or midway between them (Figure 1). If the eye-movement theory is correct, the target will always appear to move against the tracked line, but if adjacency determines the direction of induced mo-

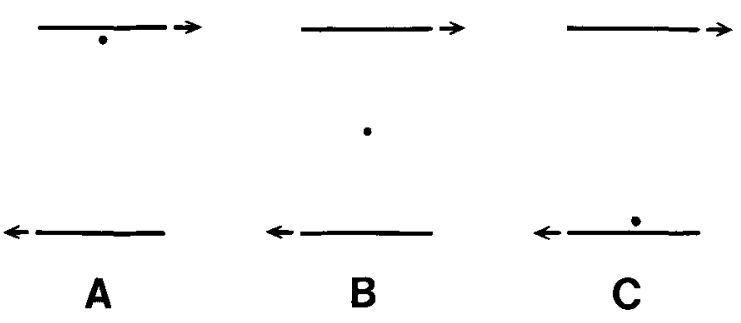

Figure 1. The stationary target was (A) adjacent to the top line, (B) midway between the lines, and (C) adjacent to the bottom line. In the tracking condition, an additional spot was placed in the middle of one of the lines and moved with it. tion, the target will always appear to move against the closer line.

\section{PROCEDURE}

There were two conditions, fixation and tracking, and the same basic display was used in both. A smoked glass screen was in front of the display, which was generated on a Tektronix oscilloscope (Model 5103N) with a fast phosphor (P31). A 2.4-deg horizontal line was placed $3.6 \mathrm{deg}$ above another 2.4-deg horizontal line. (The first two observers viewed 3.6-deg lines.) These lines were generated by a high-frequency triangle wave output from a Wavetek function generator (Model 116VCG) which acted through two separate Tektronix multiplexer (Model 470) channels. Both lines moved through two full cycles at a rate of $.48 \mathrm{deg} / \mathrm{sec}$ and were $180 \mathrm{deg}$ out of phase. Their motion was generated by the sine-wave output of another function generator (Wavetek Model 116VCG).

A stationary target was placed in the middle of the display in any of three locations: .24 deg below the upper line, midway between the two lines, or .24 deg above the lower line. In each condition, there were 5 trials with each of these three placements, or 15 trials, which were counterbalanced. Since there were two conditions, fixation and tracking, each observer viewed 30 trials.

In the fixation condition, the observer was told to fixate the stationary target, and in the tracking condition, he was told to track one of the two moving lines. To facilitate tracking, a dot was placed in the center of the tracked line and moved with it. Seven of the observers tracked the top line, six tracked the bottom line. The fixation and tracking conditions were run separately. For six subjects the fixation condition was first, and for seven subjects the tracking condition was first.

The observer's task was the same in both conditions: He was to report if the target appeared to move in the same direction as the top line or in the same direction as the bottom line. If the top line, for instance, caused the induced motion, the dot would appear to move in the same direction as the bottom line. This was a forced choice decision.

The observers viewed the display binocularly. To keep their heads still, a forehead- and chinrest were used. Two practice trials were given before each condition.

The eye movements of one observer (C.F.) were monitored with a Cornsweet-Crane eye tracker. The procedure was the same for C.F., except that a bite bar, rather than a chinrest, was used to restrain her head. Her data do not differ from those of the others and were analyzed together.

There were 13 unpaid observers who had normal or correctedto-normal acuity. C.F. had normal acuity.

\section{RESULTS AND DISCUSSION}

The results were tabulated as follows. Each observer was classified as having responses "determined" either by the top line or by the bottom line. If the apparent motion of the target was in the same direction as the bottom line in three of the five trials at a given target placement (i.e., near top line, midway, near bottom line), this observer was classified as having his response determined by the bottom line. The chi-square test was then performed on these data. (The actual frequencies are presented in parentheses.)

\section{Fixation Condition}

There were 5 trials at each of 3 target placements for 13 observers, totaling 65 trials per condition. 
It was possible to make a prediction on the basis of adjacency in the fixation condition only when the target was adjacent to one or the other line, and this prediction was correct in $93 \%$ of these trials. When the top line was adjacent, it determined the direction of induced motion for all 13 observers $\left(\chi^{2}=11.08\right.$, $p<.001)$ and did so in $95 \%$ (62 of 65 ) of these trials. When the bottom line was adjacent, it determined the direction of induced motion for all but one observer $\left(\chi^{2}=7.69, \mathrm{p}<.01\right)$ and in $91 \%$ (59 of 65) of these trials. When the target was midway between the two lines, the top line determined the direction of induced motion for 10 of 13 observers, which was not statistically significant $\left(\chi^{2}=2.77\right.$, $p<.10)$. This occurred in $65 \%(42$ of 65$)$ of the trials.

\section{Tracking Condition}

Seven observers tracked the top line, six tracked the bottom line, yet the results are nearly identical to those of the fixation condition. According to the eye-movement theory, the tracked line should have determined the direction of induced motion regardless of which line was more adjacent, but it did so in only $47 \%$ (91 of 195 ) of all trials. The target appeared to move against the top line in 53\% (56 of 105) of the trials in which the top line was tracked and against the bottom line in $39 \%$ ( 35 of 90 ) of the trials in which it was tracked. These results would be expected on the basis of chance.

It was possible to make a prediction on the basis of adjacency only when the target was adjacent to one or the other line, and this prediction was correct in $95 \%$ of these trials regardless of which line was tracked. When the top line was adjacent, it determined the direction of induced motion for all 13 observers $\left(\chi^{2}=11.08, \mathrm{p}<.001\right)$. This occurred in $97 \%$ (63 of 65 ) of all trials-in $94 \%$ of the trials in which the top line was tracked and in $100 \%$ of the trials in which the bottom line was tracked. When the bottom line was adjacent, it, too, determined the direction of induced motion for all 13 observers $\left(\chi^{2}=11.08, \mathrm{p}<.001\right)$. This occurred in $94 \%$ (61 of 65 ) of all trials-in $97 \%$ of those in which the top line was tracked and $90 \%$ of those in which the bottom line was tracked.

Among these trials, there were many instances in which the predictions from both theories were the same. If we eliminate these ambiguous trials, we are left with trials in which one line was tracked but the target was adjacent to the other line. The top line, for instance, might have been tracked while the target was adjacent to the bottom line, or vice versa. The eye-movement theory would predict that the tracked line would determine the direction of induced motion; on the basis of adjacency, the adjacent line would be expected to determine the direction of induced motion. The adjacent line did determine the direction of induced motion for all 13 observers $\left(\chi^{2}=11.08\right.$, $p<.001)$ and on all but one of these 65 trials, or in $98 \%$. In sum, the results clearly show that adjacency, not eye movements, determined the direction of induced motion in these trials.

It would be possible to argue that the effect of eye movements was overwhelmed by that of adjacency, but even when adjacency was neutralized-when the target was midway between the lines-eye movements had no effect; the tracked line determined the direction of induced motion for only 5 of 13 observers $\left(\chi^{2}=.31\right)$ and in $46 \%$ (30 of 65 ) of these trials, or at about chance level.

The one observer whose eye movements were recorded was instructed to track the top line. Her tracking was accurate, averaging $2.3 \mathrm{deg}$ (the line's excursion was $2.4 \mathrm{deg}$ ), and showed few saccades. The top line determined induced motion in each of the five trials in which it was adjacent; when the bottom line was adjacent, however, it determined the direction of induced motion in all five trials. When the target was midway between the lines, the top line determined the direction of induced motion in only three of five trials.

In conclusion, object-relative displacement, as modified by adjacency, can account for these results. Eye movements cannot account for, and do not even seem to affect, induced motion.

\section{REFERENCES}

Brosgole, L., Cristal, P. M., Carpenter, O. The role of eye movements on the perception of visually induced motion. Perception \& Psychophysics, 1968, 3, 166-168.

Bruell, J. M., \& Albee, G. W. Notes toward a motor theory of visual egocentric localization. Psychological Review, 1955, $62,391-400$.

Festinger, L., \& Easton, A. M. Inferences about the efferent system based on a perceptual illusion produced by eye movements. Psychological Review, 1974, 81, 44-58.

Festinger, L., Sedgwick, H. A., \& Holtzman, J. D. Visual perception during smooth pursuit eye movements. Vision Research, 1976, 16, 1377-1386.

GoGel, W. C. Relative motion and the adjacency principle. Quarterly Journal of Experimental Psychology, 1974, 26, 425437.

Gogel, W. C., \& Koslow, M. The effect of perceived distance on induced movement. Perception \& Psychophysics, 1971, 10, 142-146.

Gogez, W. C., \& Koslow, M. The adjacency principle and induced motion. Perception \& Psychophysics, 1972, 11, 309-314.

KaUfman, L. Sight and mind. New York: Oxford Press, 1974.

Mack, A., \& Herman, E. A new illusion: The underestimation of distance during pursuit movements. Perception \& Psychophysics, 1972, 12, 471-473.

Mack, A., \& Herman, E. Position constancy during pursuit eye movement: An investigation of the Filehne illusion. Quarterly Journal of Experimental Psychology, 1973, 25, 71-84.

(Received for publication June 6, 1979; revision accepted September 26, 1979.) 